\title{
Magnetohydrodynamic counter-rotating vortices and synergetic stabilizing effects of magnetic field and plasma flow
}

\author{
G. N. Throumoulopoulos ${ }^{1}$, H. Tasso ${ }^{2}$ \\ ${ }^{1}$ University of Ioannina, Association Euratom-Hellenic Republic, \\ Division of Theoretical Physics, GR 45110 Ioannina, Greece \\ ${ }^{2}$ Max-Planck-Institut für Plasmaphysik, Euratom Association, \\ D-85748 Garching, Germany
}

\begin{abstract}
A nonlinear two-dimensional steady state solution in the framework of hydrodynamics describing a row of periodic counter-rotating vortices is extended to the magnetohydrodynamic (MHD) equilibrium equation with incompressible flow of arbitrary direction. The extended solution covers a variety of equilibria because four surface quantities remain free. Similar to the case of the MHD cat-eyes equilibrium [Throumoulopoulos et al., J. Phys. A: Math. Theor. 42, 335501 (2009)] and unlike linear equilibria, the flow has a strong impact on isobaric surfaces by forming pressure islands located within the counter-rotating vortices even for values of $\beta$ (defined as the ratio of the thermal pressure over the external axial magnetic-field pressure) on the order of 0.01. Also, the axial current density is appreciably modified by the flow. Furthermore, a magnetic-field-aligned flow of experimental fusion relevance, i.e for Alfvén Mach numbers of the order of 0.01 , and the flow shear in combination with the variation of the magnetic field perpendicular to the magnetic surfaces have significant stabilizing effects potentially related to the equilibrium nonlinearity. The stable region is enhanced by an external axial magnetic field.
\end{abstract}

Published in Phys. Plasmas 17, 032508 (2010) 


\section{Introduction}

Sheared flows influence the equilibrium and stability properties of magnetically confined plasmas and result in transitions to improved confinement regimes of fusion devices. Typical experimental velocities corresponding to Alfvén Mach numbers, $M$, on the order of 0.01 have been observed in JET (Ref. [1]) and ASDEX Upgrade (Ref. [2]) tokamaks. Theoretically, there has been an increasing number of studies on the equilibrium and stability properties of flowing plasmas (see, for example, Refs. [3]-[21] and Refs. cited therein). In particular, analytic equilibrium solutions have been obtained as solutions to generalized Grad-Shafranov equations (Refs. $[3,4,6,7,9,10,11,13])$. An impact of the equilibrium flow related to the convective term in the momentum equation is that the isobaric surfaces deviate from the magnetic surfaces. For linear equilibria and $M \approx 0.01$ this deviation is weak (see, for example, Ref. [3]). Regarding stability, the problem becomes tough because of the same convective flow term which makes the force operator non-hermitian. The difficulty reflects to the lack of necessary and sufficient conditions for stability, an old unsolved problem even in the framework of hydrodynamics. Thus, only certain sufficient conditions have been obtained (Refs $[14,15,18,19,20,21])$. In connection with the present study we mention a readily applicable sufficient condition for linear stability pertinent to plasmas of constant density and parallel incompressible flows (Ref. [21]) (see also Sec. III).

Motivation of the present study was a previous paper (Ref. [13]) in which the cat-eyes hydrodynamic equilibrium solution describing a row of identical vortices was extended to magnetohydrodynamic plasmas with incompressible flow. In this study it was found that for flows with $M \approx 0.01$ the pressure surfaces deviate strongly from magnetic surfaces by forming pressure islands in the location of the cat eyes, unlike the weak respective deviation in linear equilibria. Thus, this strong flow effect should be related to the equilibrium nonlinearity. In addition, a flow parallel to the magnetic field results in appreciable stabilizing effects.

Here we examine the validity of these conclusions in the case of another nonlinear equilibrium solution which in the framework of hydrodynamics describes a row of counter-rotating vortices (Ref. [22]) by extending this solution to MHD for plasmas with incompressible flow of arbitrary direction. The extended solution may be more relevant to laboratory fusion plasmas than cat-eyes because it can be bounded. The impact of flow on the pressure 
is then examined. Also, for parallel flows the stability is studied by applying the aforementioned sufficient condition (Ref. [21]). Conclusions similar to those in the case of cat-eyes are drawn as concerns the formation of pressure islands and stabilizing effects of flow. Pressure islands, however, are not necessary for stabilization for large values of $\beta$ on the order of 0.1. Owing to the objectives, the study is conducted along the same lines as in Ref. [13] including a couple of additional issues: the impact of flow on the axial current density and the role of the individual terms of a quantity $A$ involved in the stability condition [Eqs. (14-19) of Sec. III]. The latter decomposition shows that stabilization is caused by a combination of flow and the magnetic field variation perpendicular to the magnetic surfaces.

For convenience the study will be presented in a self contained way because otherwise repeated reference to [13] would make reading tedious. In Sec. II the extended counter-rotating-vortices equilibrium is derived by solving the pertinent generalized Grad-Shafranov equation. Also the impact of flow on the pressure and axial current density is examined. Then, the stability condition is applied to the solution constructed for a broad variation of the free parameters in Sec. III. The impact of the magnetic surface shaping, amplitude and shear of the flow, thermal pressure and axial magnetic field on the stable region is also examined. Section IV summarizes the conclusions. 


\section{MHD counter-rotating-vortices equilibrium with flow}

We consider a translational symmetric magnetized plasma with incompressible flow whose equilibrium states satisfy the generalized Grad-Shafranov equation (Refs. [6, 9]),

$$
\left(1-M^{2}\right) \nabla^{2} \psi-\frac{1}{2}\left(M^{2}\right)^{\prime}|\nabla \psi|^{2}+\left(\mu_{0} P_{\mathrm{s}}+\frac{B_{z}^{2}}{2}\right)^{\prime}=0
$$

and the Bernoulli relation for the pressure

$$
P=P_{\mathrm{s}}(\psi)-\frac{1}{2 \mu_{0}} M^{2}|\nabla \psi|^{2} .
$$

Here, $\psi(x, y)$ is the poloidal magnetic flux function which labels the magnetic surfaces; $(x, y, z)$ are Cartesian coordinates with $z$ corresponding to the axis of symmetry and $(x, y)$ associated with the poloidal plane; $M(\psi)$ the Mach function of the poloidal velocity with respect to the poloidal-magnetic-field Alfvén velocity; $B_{z}$ the axial magnetic field; for vanishing flow the surface function $P_{\mathrm{s}}(\psi)$ coincides with the pressure; the prime denotes a derivative with respect to $\psi$. The surface quantities $M(\psi), B_{z}(\psi)$ and $P_{\mathrm{s}}(\psi)$ are free functions for each choice of which (1) is fully determined and can be solved whence the boundary condition for $\psi$ is given. Also, to completely determine the equilibrium, three additional surface functions are needed, i.e, the density, $\varrho(\psi)$, the electrostatic potential, $\Phi(\psi)$, and the axial velocity component $v_{z}(\psi)$. Details including derivation of (1) and (2) can be found in Refs. $[7,9]$.

Equation (1) can be simplified by the transformation (Refs. [23, 24])

$$
u(\psi)=\int_{0}^{\psi}\left[1-M^{2}(g)\right]^{1 / 2} d g,
$$

which reduces (1) to

$$
\nabla^{2} u+\frac{d}{d u}\left(\mu_{0} P_{\mathrm{s}}+\frac{B_{z}^{2}}{2}\right)=0
$$

Also, (2) is put in the form

$$
P=P_{\mathrm{s}}(u)-\frac{M^{2}}{2 \mu_{0}\left(1-M^{2}\right)}|\nabla u|^{2} .
$$


Note that (4) free of a quadratic term as $|\nabla u|^{2}$ is identical in form with the quasistatic [25] MHD equilibrium equation as well as to the equation governing the steady motion of an inviscid incompressible fluid in the framework of hydrodynamics. Transformation (3) does not affect the magnetic surfaces, it just relabels them. Also, once a solution of (4) is found, the equilibrium can be completely constructed in the $u$-space; in particular, the magnetic field, current density, velocity, and electric field can be determined by relations (9)-(12) of Ref. [13].

To construct the extended counter-rotating-vortices solution to (4) we first introduce for convenience dimensionless quantities: $\tilde{x}=x / L, \tilde{y}=y / L$, $\tilde{u}=u /\left(B_{z 0} L\right), \tilde{\varrho}=\varrho / \varrho_{0}, \tilde{P}=P /\left(B_{z 0}^{2} / \mu_{0}\right), \tilde{\mathbf{B}}=\mathbf{B} / B_{z 0}, \tilde{\mathbf{j}}=\mathbf{j} /\left(B_{z 0} /\left(\mu_{0} L\right)\right)$, $\tilde{\mathbf{v}}=\mathbf{v} / v_{A 0}$, where $v_{A 0}=B_{z 0} / \sqrt{\mu_{0} \varrho_{0}}$, and $\tilde{\mathbf{E}}=\mathbf{E} /\left(B_{z 0} v_{A 0}\right)$; here, $L, B_{z 0}$, and $\varrho_{0}$ are reference quantities to be defined later. Equations (4) and (5) hold in identical forms for the tilted quantities and will be further employed as dimensionless by dropping for simplicity the tilde. Then, we make the ansatz

$$
\frac{d\left(P_{\mathrm{s}}+B_{z}^{2} / 2\right)}{d u}=\frac{1-\epsilon^{2}}{2} \sinh (2 u),
$$

by which (4) reduces to the following form of sinh-Poisson equation:

$$
\nabla^{2} u=-\frac{1-\epsilon^{2}}{2} \sinh (2 u)
$$

Equation (7) admits the solution

$$
u=-2 \operatorname{arctanh}\left(\frac{\epsilon \cos (x)}{\cosh (\epsilon y)}\right)
$$

the characteristic lines of which are shown in Fig. 1. The configuration consists of an infinite series of periodic pairs of vortices having magnetic axes on $(x=k \pi, y=0)$ with $k$ an integer. Also, it holds $u(x=k \pi+\pi / 2)=0$ and therefore boundary conditions at the points $x=k \pi+\pi / 2$ can be readily imposed (see Fig. 1). The velocity of the individual vortices of each pair have opposite direction. The magnetic field and current density lie on the velocity or magnetic surfaces and therefore the vortices can be regarded as magnetic islands with plasma flow. Quasistatic MHD and hydrodynamic counter-rotating vortices can be recovered as particular cases. The magnetic surface elongation along $y$ gets shorter as the parameter $\epsilon$ increases with $\epsilon= \pm 1$ corresponding to point vortices. In comparison with the cat-eyes 
equilibrium [Eq. (15) of Ref. [21]] the extended counter-rotating vortices have the following diverse characteristics:

1. Solution (8) is inherently two-dimensional (for $0<|\epsilon| \leq 1$ ) while the cat-eyes solution for $\epsilon=0$ becomes one-dimensional (note that here $u(\epsilon=0) \equiv 0)$.

2. Except for the bounding surfaces, $u=0$, the configuration has only closed magnetic surfaces while cat eyes have a separatrix.

3. It holds $\lim u_{y \rightarrow \infty}=0$, unlike the cat-eyes solution which in this limit becomes singular.

Equation (6) can be solved for $P_{\mathrm{s}}(u)+B_{z}(u)^{2} / 2$ to yield

$$
\begin{aligned}
P_{\mathrm{s}}+\frac{B_{z}^{2}}{2} & =\frac{1-\epsilon^{2}}{4} \cosh (2 u)+c_{0} \\
& =\frac{1-\epsilon^{2}}{4} \cosh \left[4 \operatorname{arctanh}\left(\frac{\epsilon \cos (x)}{\cosh (\epsilon y)}\right)\right]+c_{0},
\end{aligned}
$$

where $c_{0}$ is a constant. In fact, (8) and (9) hold for a rather large set of equilibria because the functions $\rho(u), \Phi(u), M(u)$ and one out of $B_{z}(u)$ and $P_{\mathrm{s}}(u)$ remain free.

We will further consider a subset of steady sates by assigning the free functions $P_{\mathrm{s}}, B_{z}$ and $M$ as

$$
\begin{gathered}
P_{\mathrm{s}}(u)=\gamma \frac{1-\epsilon^{2}}{2}[\cosh (2 u)-1]+\frac{\beta_{\mathrm{f}}}{2}, \\
B_{z}^{2}(u)=(1-2 \gamma) \frac{1-\epsilon^{2}}{2} \cosh (2 u)+B_{z 0}^{2}, \\
M=M_{0}[\cosh (-2 u)-1]^{n}, \quad n>0 .
\end{gathered}
$$

Choice (12) yields a peaked $M^{2}$-profile along $y$ with $\left|M_{0}\right|$ being the maximum absolute value at $x=y=0$ and $\lim M_{y \rightarrow \infty}^{2}=0$. The profile becomes steeper as $n$ takes larger positive values, thus increasing the shear of $M$ in relation to the velocity shear. The parameter $B_{z 0}$ represents the external axial magnetic field,

$$
\beta \equiv \frac{P_{\mathrm{s}}(x=y=0)}{\left(B_{z 0}^{2} / 2\right)}=\gamma\left(1-\epsilon^{2}\right)[\cosh (4 \operatorname{arctanh}(\epsilon))-1]
$$


and $\beta_{\mathrm{f}}=P_{\mathrm{s} 0} /\left(B_{z 0}^{2} / 2\right)$, where $P_{\mathrm{s} 0}=$ const. Note that $\gamma$ has been introduced in (10) and (11) in such a way that (9) is automatically satisfied. The physical parameter $\beta$ (the ratio of thermal pressure on axis over the $B_{z 0}$-pressure) is related to $\gamma$. The other parameter $\beta_{\mathrm{f}}$ in (10) yields force-free quasistatic equilibria when $\gamma=\beta=0$. For $\beta \neq 0$, we set $\beta_{\mathrm{f}}=0$ in order that $P_{\mathrm{s}}$ vanishes for $y \rightarrow \infty$; thus, only one of the parameters $\beta$ and $\beta_{\mathrm{f}}$ is finite in connection with peaked and flat $P_{\mathrm{s}}$-profiles, respectively. A peaked $P_{s}$-profile is shown in Fig. 2. The parameters $M_{0}$, and $n$ are free together with $L, \varrho_{0}, \epsilon, B_{z 0}$, and $\beta$ or $\beta_{\mathrm{f}}$. It is recalled that dimensionless quantities are employed and therefore $B_{z 0}=1$. Also, the reference quantities $L$ and $\varrho_{0}$ not appearing explicitly in the equations can arbitrarily be defined as the vortex length (along the $x$-axis) and the density at $x=y=0$. Because of the many free parameters there is a variety of steady states. An example for $\gamma=1 / 2$ is an equilibrium with peaked $P$-profile shown in Fig $4(\mathrm{a}), B_{z}=B_{z 0}$, axial current density and three-component velocity. Another example for $\beta=M_{0}=0$ is a force free quasistatic equilibrium with three-component current density. In the presence of flow, however, the pressure profile becomes hollow [see Fig. $4(\mathrm{~b})]$.

We have examined the pressure and current density by Mathematica 6 within broad regions of the free parameters, i.e., $0<\epsilon \leq 1,0 \leq \beta \leq 0.9$, $0 \leq\left|M_{0}\right| \leq 0.9$ and $0 \leq n \leq 10$. Note that, because of the flow term in (5) the pressure for certain parametric values can become negative. Thus, particular care has been taken in getting everywhere physically acceptable pressure. It turns out that, as in the case of cat-eyes (Ref. [21]), the flow has strong impact on the isobaric surfaces by creating pressure island within the counter-rotating vortices. This is shown in Fig. 3. The pressure islands are twisted at an angle of $\pi / 2$ with respect to the islands of the cat-eyes equilibrium (see Fig. 3 of Ref. [21]). Also P-profiles are presented in Fig. 4. As can be seen in Fig. 3 pressure islands appear even for parametric values of experimental fusion concern $\left(\beta=0.01, M_{0}=0.02\right)$. Usual equilibria with nested pressure surfaces are also possible for appropriately large values of $\beta$ on the order of 0.1 because then the first quasistatic term in (5) dominates over the second flow term. In addition, the flow affects appreciably the axial current density, $j_{z}$, regardless of $\beta$, while the poloidal current density remains nearly unaffected. An example is shown in Fig. 5. Since for fusion pertinent linear equilibria the flow impact on the pressure and the current density is weak, it is the nonlinearity here which should play an important role. 


\section{Combined stabilizing effects of flow and magnetic field}

The linear stability of the equilibria described by (8) and (10)-(12) is now examined by applying a sufficient condition (Ref. [21]). This condition states that a general steady state of a plasma of constant density and incompressible flow parallel to $\mathbf{B}$ is linearly stable to small three-dimensional perturbations if the flow is sub-Alfvénic $\left(M^{2}<1\right)$ and $A \geq 0$, where $A$ is given by (22) of Ref. [21] (with $\lambda$ therein corresponding to $M$ here). Consequently, we restrict the study to parallel flows and set $\varrho=1$. In fact if the density is uniform at equilibrium it remains so at the perturbed state because of incompressibility (Ref. [26]). First it is noted that on the basis of Mercier expansions it turns out that the condition is never satisfied in the vicinity of the magnetic axis $(A<0)$ (Ref. [27]). This holds for generic two-dimensional equilibria irrespective of the geometry. Also, for the pressure (10), the quantity $A$ is independent of $\beta_{\mathrm{f}}$, as may be expected on physical grounds, because $A$ contains $d P_{\mathrm{s}} / d u$ and not $P_{\mathrm{s}}$ itself. It is recalled here that $\beta=0$ when $\beta_{\mathrm{f}} \neq 0$. In the $u$-space for translational symmetric equilibria, $A$ assumes the form

$$
\begin{aligned}
A & =A_{1}+A_{2}+A_{3}+A_{4}, \\
A_{1} & =-(\mathbf{j} \times \nabla u)^{2} \\
A_{2} & =(\mathbf{j} \times \nabla u) \cdot(\boldsymbol{\nabla} u \cdot \nabla) \mathbf{B} \\
A_{3} & =-\frac{1}{2} \frac{d M^{2}}{d u}\left(1-M^{2}\right)^{-1}|\nabla u|^{2} \nabla u \cdot \frac{\boldsymbol{\nabla} B^{2}}{2}, \\
A_{4} & =-\frac{1}{2} \frac{d M^{2}}{d u}\left(1-M^{2}\right)^{-3 / 2}|\nabla u|^{4} g \\
g & =\left(1-M^{2}\right)^{-1 / 2}\left(\frac{d P_{\mathrm{s}}}{d u}-\frac{d M^{2}}{d u} \frac{B^{2}}{2}\right)
\end{aligned}
$$

and $\mathbf{B}$ and $\mathbf{j}$ as given by (9) and (10) of Ref. [13]. To calculate $A$ analytically for the equilibria under consideration we developed a code in Mathematica 6. The expressions obtained for both peaked and flat $P_{\mathrm{s}}$-profiles being lengthy are not given explicitly here except for the case of quasistatic equilibria [Eq. (20) below]. The calculations led to the following conclusions. 
1. For quasistatic equilibria $\left(M_{0}=0\right)$ the quantity $A$ assumes the concise form

$$
\begin{aligned}
A= & \frac{C}{D}, \\
C= & 128 \epsilon^{6}\left(\epsilon^{2}-1\right) \cos ^{2}(x) \cosh ^{2}(2 \epsilon y)[\cos (2 x)-\cosh (2 \epsilon y)] \\
& \times\left[1+\epsilon^{2}+\epsilon^{2} \cos (2 x)+\cosh (2 \epsilon y)\right] \\
D= & {\left[\epsilon^{2}+\epsilon^{2} \cos (2 x)-\cosh (2 \epsilon y-1)\right]^{5} . }
\end{aligned}
$$

Note that $A$ becomes independent of $\beta$ and $B_{z 0}$. The condition is nowhere satisfied except for point vortices $(\epsilon=1)$, the magnetic axes, the bounding surfaces $x=k \pi+\pi / 2$ and for $y \rightarrow \infty$ for which $A=0$. A profile of $A$ is given in Fig. 6.

2. The flow results in the formation of a stable region close to the magnetic axis. An example shown the sign of $A$ on the poloidal plane is presented in Fig. 7. The red (lighter) colored regions are stable $(A \geq 0)$, while in the blue (darker) colored region it holds $A<0$. The whole area of Fig. 7 becomes blue (lighter) colored when $M_{0}=0$. Profiles of the individual four terms contained in $A$ [Eqs. (15-18)] are given in Fig. 8. As can be seen there stabilization is caused by a synergism of the fourth flow term, $A_{4}$, containing $g$ and the second term, $A_{2}$, containing $(\boldsymbol{\nabla} u \cdot \nabla) \mathbf{B}$ and therefore related to the variation of $\mathbf{B}$ perpendicular to the magnetic surfaces. Note that $A_{2}$ itself can not make the condition satisfied. The first destabilizing term, $A_{1}$ potentially relates to current driven modes. The other flow term $A_{3}$ with indefinite sign is at least two orders of magnitude lower than the other terms. It may be noted here that certain synergetic action of the magnetic field and flow for the formation of internal transport barriers in terms of reversed magnetic shear and flow shear was found theoretically in Refs. [11, 12] and observed recently in JET (Ref. [29]). Further physical understanding in the context of the present study is difficult even in the quasistatic case because we can not compare with the conventional stability theory, e.g. Mercier criterion. The reason is that $A \geq 0$ is a local condition stemming from the requirement that the integrand of inequality (12) of Ref. [21] should be positive while Mercier modes are localized in the vicinity of a magnetic surface. This difference reflects to the fact that 
the stable regions determined by $A \geq 0$ are not located near a magnetic surface.

3. The stable region broadens when the parameters $M_{0}, n$ and $\epsilon$ take larger values as can be seen in Figs. 9(a), 9(b) and 9(c), respectively. Note the sensitiveness of $A$ in the region of the stable window to the small variation of these parameters possibly related to the nonlinearity; in particular, $\epsilon$ appears in the arguments of the counter-rotating-vortices solution (8). The impact of the magnetic surface elongation on stability in connection with $\epsilon$ is opposite to that in the cat-eyes equilibrium. These results hold for both peaked- and flat- $P_{\mathrm{s}}$ equilibrium profiles. Unlikely, the stable region is rather insensitive to the variation of $\beta$. An example is given in Fig. 9(d), where the stable window persists (just getting slightly smaller) when $\beta$ is increased by an order of magnitude (from 0.01 to 0.1 ). Also, for point vortices $(\epsilon=1) A$ becomes independent of $\beta$ irrespective of the value of $M_{0}$. It should be noted that stabilization is possible for usual nested pressure surfaces for appropriately large values of $\beta$. An example is the equilibrium with $\beta=0.1$ Fig. 9(d) refers to. Thus, pending on the value of $\beta$ flow stabilization is possible for either equilibria having pressure islands or nested isobaric surfaces. This holds also for the extended cat-eyes equilibrium a result not noticed in Ref. [13]. On the other side the modification of the axial current density profile by the flow persists regardless of the values of $\beta$.

4. Although for $M_{0}=0$ the vacuum magnetic field $B_{z 0}$ has no impact on $A$ [equation (20)], in combination with the flow, $B_{z 0}$ can enhance the stable region. An example of this synergetic effect is shown in Fig. 10(a). Another example of such a strong synergism can be seen in Fig. 10(b). In this case, while the flow itself can not make $A$ positive, together with $B_{z 0}$ it results in the formation of the stable window.

\section{Conclusions}

We have constructed a counter-rotating-vortices equilibrium for MHD plasmas with incompressible flow by extending the respective hydrodynamic solution. The extended solution describes a two-dimensional non-linear equilibrium consisting of a row of periodic island pairs with counter currents and covers a variety of equilibrium configurations because four surface functions 
remain free. Compared with the extended MHD cat-eyes (Ref. [13]), the counter-rotating vortices solution may be more appropriate to magnetically confined plasmas because the plasma can be bounded.

By means of the equilibrium constructed and a criterion for linear stability we came to the following conclusions.

1. As in the case of cat-eyes, fusion pertinent flows with $M \approx 0.01$ in equilibria with $\beta \approx 0.01$ affect drastically the pressure surfaces by forming pressure islands within the counter rotating vortices. The pressure islands are twisted at an angle of $\pi / 2$ with respect to the cat-eyes islands. In addition, the axial current density is appreciably modified by the flow irrespective of $\beta$. Since such strong flow-caused changes do not occur in linear equilibria it is the equilibrium nonlinearity here which plays an important role.

2. A parallel flow and the flow shear in conjunction with the variation of B perpendicular to the magnetic surfaces have remarkable stabilizing effects potentially correlated to the equilibrium nonlinearity. Note that in the case of a linear sinusoidal solution describing a tokamak equilibrium with parallel incompressible flow in toroidal geometry (Ref. [28]), the stability condition is not satisfied for $M \approx 0.01$ because the stabilizing flow term, $A_{4}$ [see Eq. (18)] becomes three orders of magnitude lower than in the present study. In both the extended cat-eyes and counter-rotating-vortices equilibria, stabilization is also possible for nested pressure surfaces in connection with large values of $\beta$ on the order of 0.1 ; in this respect the flow-caused change of the equilibrium axial current density being independent of $\beta$ might play a more important role on stability.

3. The stable regions shorten with the magnetic surface elongation along the non periodic direction, broaden with the flow and the flow shear and are rather insensitive to thermal pressure. In addition, a combination of flow and a constant axial magnetic field have synergetic stabilizing effects by enlarging the stable region.

The generic validity of the results of the present and cat-eyes studies could be further examined by alternative non-linear solutions. Since in plane geometry the Laplace operator is involved in the equilibrium equation, such solutions can be constructed by using the powerful tool of complex functions. 
Also, it is interesting to examine the impact of toroidicity by extending the study to toroidal geometry. Because of unavailability of analytic nonlinear solutions in this case, most likely the construction should be performed numerically. Finally, it is noted that in Ref. [28] only linear low-shear Mach functions were considered. Therefore, to check the importance of the equilibrium nonlinearity on stability, the potential role of the flow shear could be further examined in the linear regime by employing Mach functions of the form $M \propto u^{n}$.

\section{Acknowledgements}

Part of this work was conducted during a visit of G.N.T. to the Max-PlanckInstitut für Plasmaphysik, Garching. The hospitality of that Institute is greatly appreciated.

The work leading to this article was performed within the participation of the University of Ioannina in the Association Euratom-Hellenic Republic, which is supported in part by the European Union (Contract of Association ERB 5005 CT 99 0100) and by the General Secretariat of Research and Technology of Greece. The views and opinions expressed herein do not necessarily reflect those of the European Commission. 


\section{References}

[1] P. C. de Vries, K. M. Rantamäki, C. Giroud E. Asp, G. Corrigan, A. Eriksson, M. de Greef, I. Jenkins, H. C. M. Knoops, P. Mantica H. Nordman, P. Strand, T. Tala, J. Weiland, K-D Zastrow and JET EFDA Contributors, Plasma Phys. Control. Fusion 48 1693, 2006.

[2] S. Günter , R. C. Wolf , F. Leuterer, O. Gruber, M. Kaufmann, K. Lackner, M. Maraschek, P. J. McCarthy, H. Meister, A. Peeters, G. Pereverzev, H. Salzmann, S. Schade, J. Schweinzer, W. Suttrop, and the ASDEX Upgade Team, Phys. Rev. Lett. 84, 3097 (2000).

[3] E. K. Maschke and H. Perrin, Plasma Physics 22 579, 1980.

[4] R. A. Clemente and R. Farengo, Phys. Fluids 27 776, (1984).

[5] A. Lifschitz and J. P. Goedbloed, J. Plasma Physics 5861 (1997).

[6] G. N. Throumoulopoulos, H. Tasso, Phys. Plasmas 4, 1492 (1997).

[7] H. Tasso, G. N. Throumoulopoulos, Phys. Plasmas 5, 2378 (1998).

[8] R. Betti, J. P. Freidberg, Phys. Plasmas 7, 2439 (2000).

[9] Ch. Simintzis, G. N. Throumoulopoulos, G. Pantis, H. Tasso, Phys. Plasmas 8, 2641 (2001).

[10] Oleg. I. Bogoyavlenskij, Physical Review E 66, 056410 (2002).

[11] G. Poulipoulis, G. N. Throumoulopoulos and H. Tasso, Plasma Phys. Control. Fusion 46, 639 (2004).

[12] G. Poulipoulis, G. N. Throumoulopoulos and H. Tasso, J. Plasma Physics 73, 347 (2007).

[13] G. N. Throumoulopoulos, H. Tasso and G. Poulipoulis, J. Phys. A: Math. Theor. 42, 335501 (2009).

[14] S. Friedlander, M. M. Vishik, Chaos 5, 416 (1995).

[15] H. Tasso, Phys. Lett. A 222, 97 (1996).

[16] E. Hameiri, Phys. Plasmas 5, 3270 (1998). 
[17] A. V. Kats JETP Letters 77, 657 (2003).

[18] K. I. Ilin and V. A. Vladimirov, Phys. Plasmas 11, 3586 (2004).

[19] M. Hirota, Z. Yoshida, E. Hameiri Phys. Plasmas 13, 022107 (2006).

[20] V. I. Ilgisonis, I. V. Khalzov and A. I. Smolyakov, Nucl. Fusion 49, 035008 (2009).

[21] G. N. Throumoulopoulos, H. Tasso, Phys. Plasmas 14, 122104 (2007).

[22] R. Mallier and S. A. Maslowe, Phys. Fluids A 5, 1074 (1993).

[23] R. A. Clemente, Nucl. Fusion 33, 963 (1993).

[24] P. J. Morrison, private communication: this transformation was discussed in a talk entitled "A generalized energy principle" and delivered in the Plasma Physics Division Meeting of the APS, Baltimore, 1986.

[25] The term quasistatic has the meaning that the velocity term is neglected in the momentum equation but is kept in Ohm's law.

[26] H. Tasso, G. N. Throumoulopoulos, On MHD stability of gravitating plasmas with field aligned flows, Report http://arxiv.org/arXiv:0905.3976v1 (2009), submitted to J. Plasma Physics.

[27] Since the condition is sufficient, when not satisfied $(A<0)$ it becomes indecisive.

[28] D. Apostolaki, G. N. Throumoulopoulos, H. Tasso, $35^{\text {th }}$ EPS Confernece on Plasma Phys., 9-13 June 2008, Hersonissos, Greece, ECA Vol. 32, P2-057 (2008).

[29] P. C. de Vries, E. Joffrin, M. Brix, C. D. Challis, K. Crombé, B. Esprosito, N. C. Hawkes, C. Giroud, J. Hobirk, J. Lönnroth, P. Mantica, D. Strintzi, T. Tala, I. Voitsekhovitch and LET-EFDA Contributors to the Work Programme, Nucl. Fusion 49, 075007 (2009). 


\section{Figure captions}

Figure 1: $u$-lines of the MHD counter-rotating-vortices solution (8) for $\epsilon=$ 0.4 as intersections of the magnetic surfaces with the poloidal plane.

Figure 2: Profile of the quasistatic pressure function $P_{\mathrm{s}}$ [equation (10)] along the $y$-axis.

Figure 3: Pressure islands in connection with (5) and (10). The curves represent pressure lines on the poloidal plane. In the absence of flow the lines of Fig. (a) coincide with the $u$-lines of Fig. 1 while the equilibrium of Fig. (b) becomes force-free.

Figure 4: Pressure profiles along the $y$-axis respective to the pressure-island configurations 3(a) and 3(b). For vanishing flow profile (a) reduces to the $P_{\mathrm{s}}$-profile of Fig. 2 and profile (b) becomes flat.

Figure 5 (Color on line): Profiles of the axial current density $j_{z}$.

Figure 6: Profile of the quantity $A$ [Eqs. (14-19)] associated with the sufficient condition for linear stability for a quasistatic equilibrium $\left(M_{0}=0\right)$. Except for the marginally stable points $y=0$ and $y \rightarrow \infty$ the condition is nowhere else satisfied.

Figure 7(Color on line): Stabilization effect of flow: In the presence of flow the red (lighter) colored stable regions appear in the diagram (a) where $A \geq 0$. The respective stable window can be seen in the profile of $A$ in (b).

Figure 8: Profiles of the four individual terms the stability quantity $A$ comprises of [Eqs. (14)-(19)] for $\epsilon=0.4, \beta=0.01, M_{0}=0.02$ and $n=6$. Stabilization is caused by combination of the term $A_{2}$ related to the variation of the magnetic field perpendicular to the magnetic surfaces and the flow term $A_{4}$.

Figure 9(Color on line): Impact of the flow (a), flow shear (b), island size (c) and thermal pressure (d) in connection with a variation of the parameters $M_{0}, n, \epsilon$, and $\beta$, respectively, on the flow caused stable window associated with $A \geq 0$ for the equilibrium of Fig. 3(a).

Figure 10(Color on line): Combined stabilization effect of flow and $B_{z 0}$ : The curve (a) indicates a stabilizing synergism of $B_{z 0}$ and flow for the equilibrium of Fig. 3(a). A stronger synergism of this kind is shown in Fig. 3(b) in which 
the stability window can not appear by the sole presence of flow. 


\section{List of Figures}

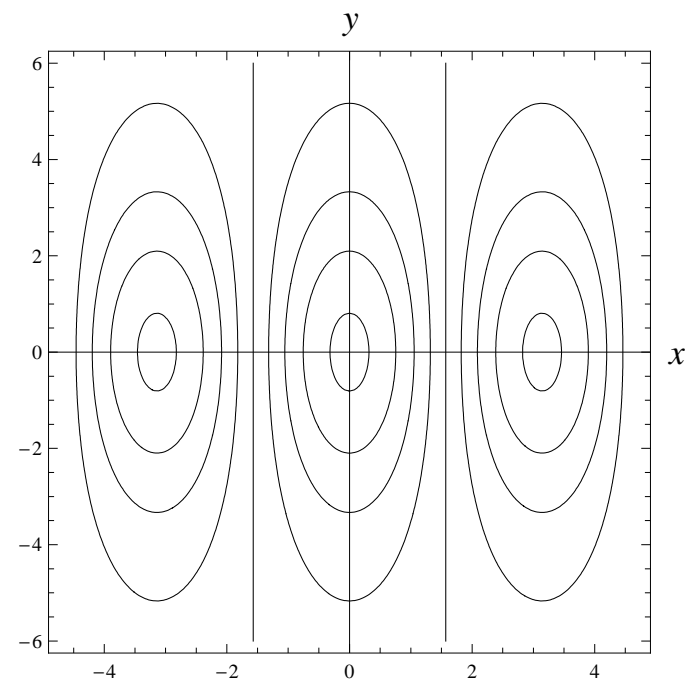

Figure 1: $u$-lines of the MHD counter-rotating-vortices solution (8) for $\epsilon=0.4$ as intersections of the magnetic surfaces with the poloidal plane.

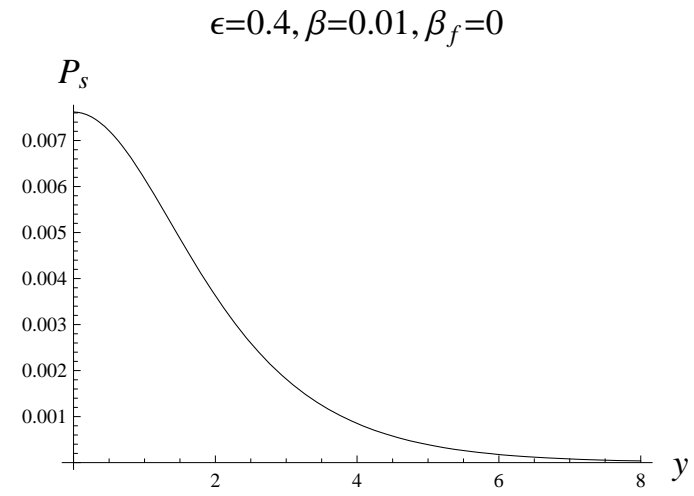

Figure 2: Profile of the quasistatic pressure function $P_{\mathrm{s}}$ [equation (10)] along the $y$-axis. 
(a) $\epsilon=0.4, \beta=0.01, M_{0}=0.02, \mathrm{n}=6$

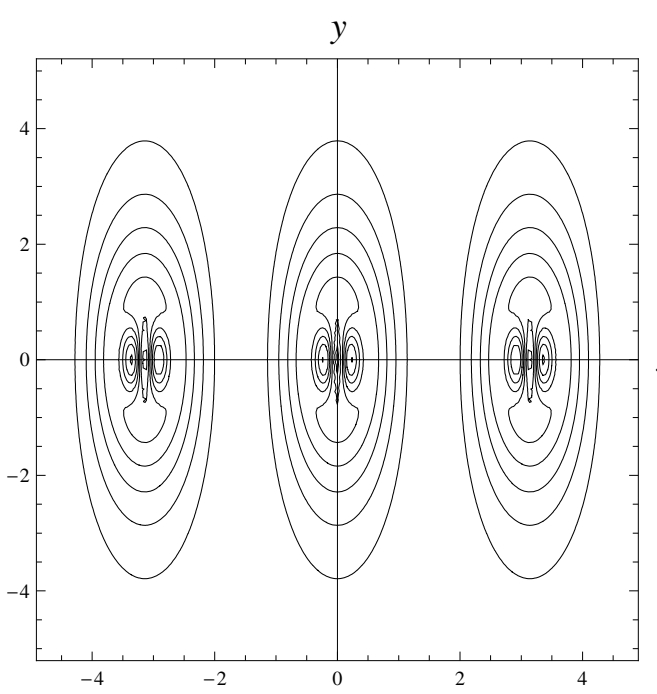

(b) $\epsilon=0.4, \beta_{f}=0.02, M_{0}=0.02, \mathrm{n}=6$

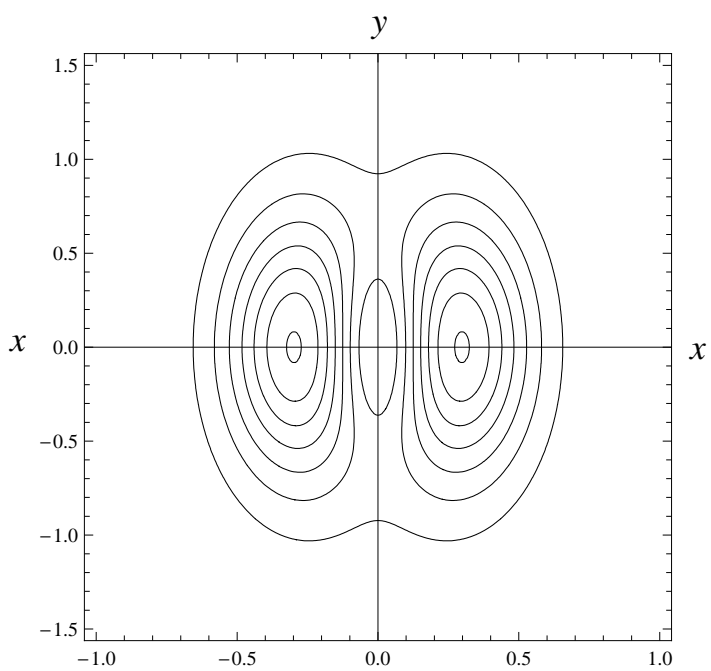

Figure 3: Pressure islands in connection with (5) and (10). The curves represent pressure lines on the poloidal plane. In the absence of flow the lines of Fig. (a) coincide with the $u$-lines of Fig. 1 while the equilibrium of Fig. (b) becomes force-free.

(a) $\epsilon=0.4, \beta=0.01, \beta_{f}=0$

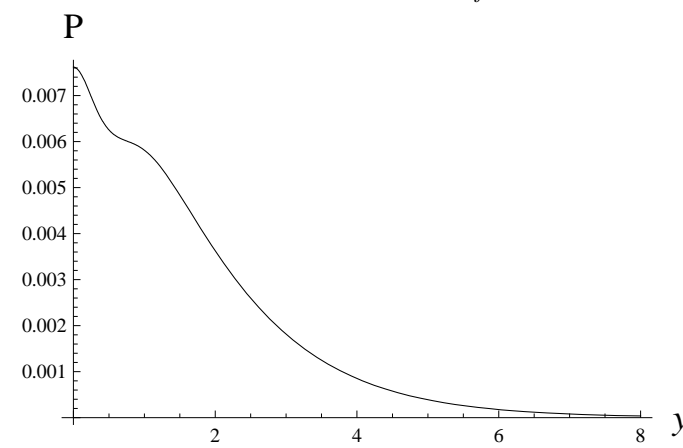

(b) $\epsilon=0.4, \beta_{f}=0.01, \beta=0$

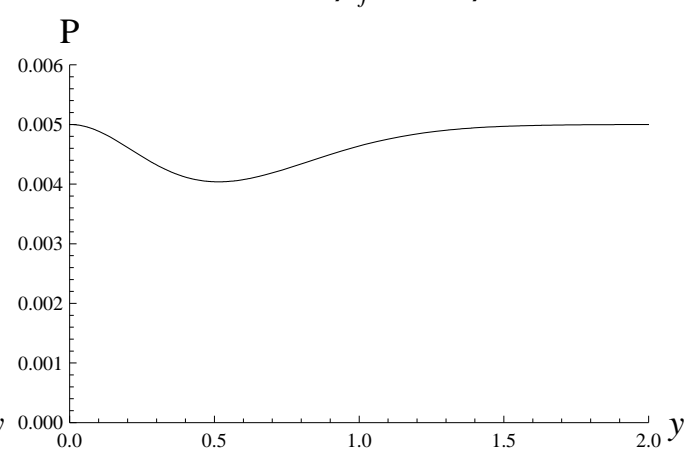

Figure 4: Pressure profiles along the $y$-axis respective to the pressure-island configurations 3(a) and 3(b). For vanishing flow profile (a) reduces to the $P_{\mathrm{s}}$-profile of Fig. 2 and profile (b) becomes flat. 
(a) $\epsilon=0.4, \beta=0.01, \mathrm{n}=6$

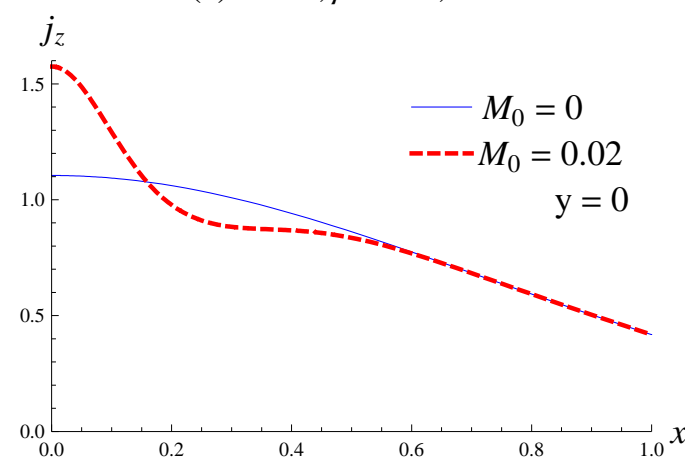

(b) $\epsilon=0.4, \beta=0.01, \mathrm{n}=6$

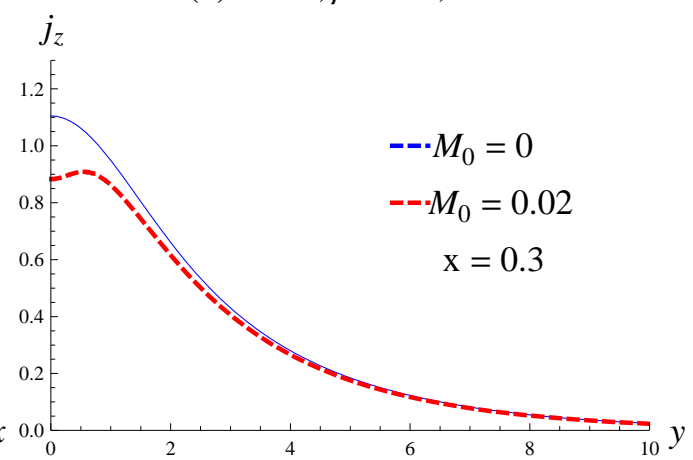

Figure 5: (Color on line) Profiles of the axial current density

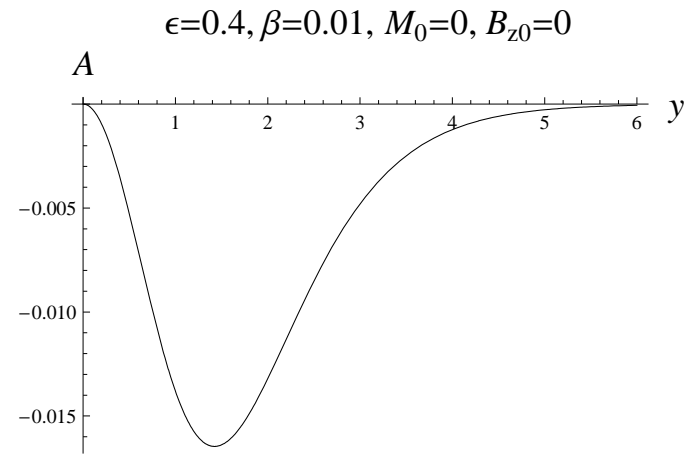

Figure 6: Profile of the quantity $A$ [Eqs. (14-19)] associated with the sufficient condition for linear stability for a quasistatic equilibrium $\left(M_{0}=0\right)$. Except for the marginally stable points $y=0$ and $y \rightarrow \infty$ the condition is nowhere else satisfied. 
(a) $\epsilon=0.4, \beta=0.01, M_{0}=0.02, \mathrm{n}=6$

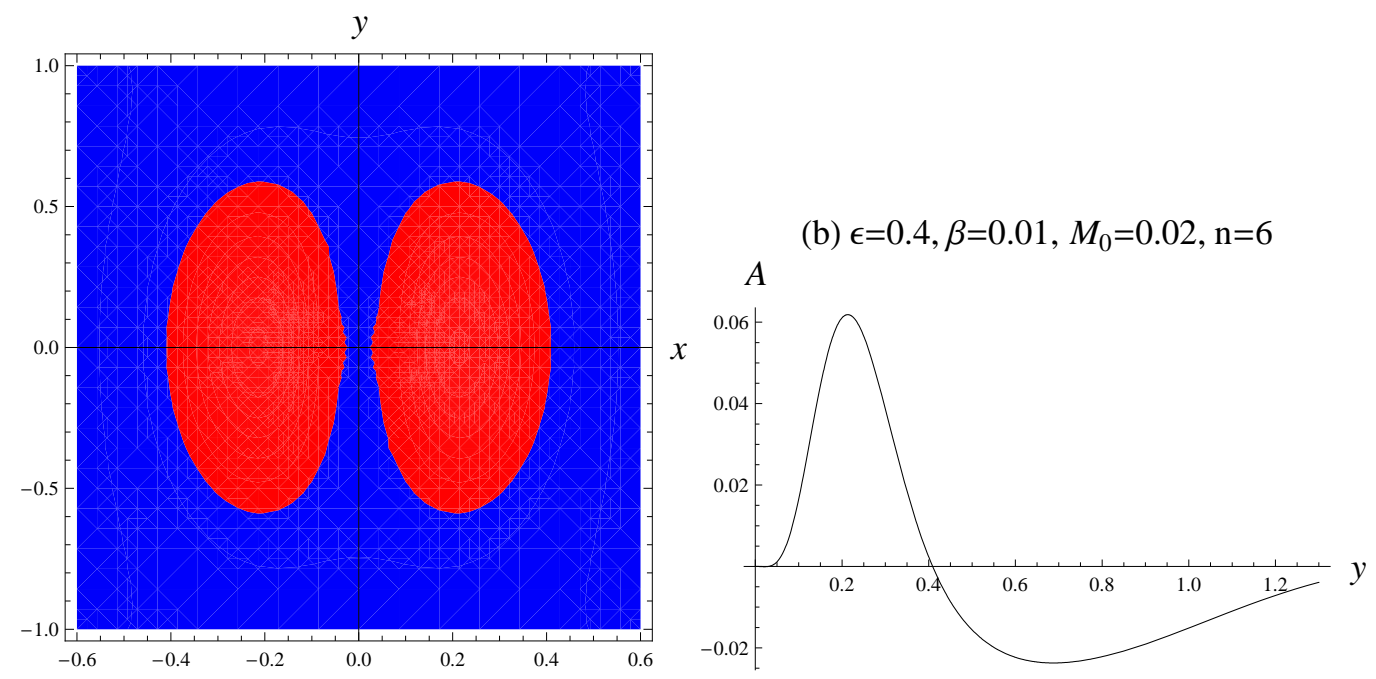

Figure 7: (Color on line) Stabilization effect of flow: In the presence of flow the red (lighter) colored stable regions appear in the diagram (a) where $A \geq 0$. The respective stable window can be seen in the profile of $A$ in (b). 
(a)

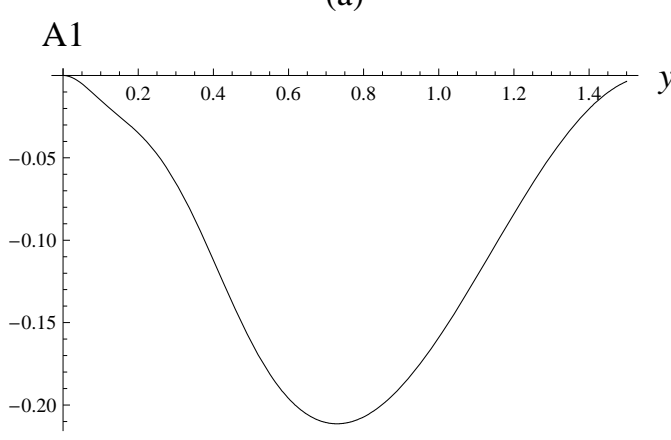

(c) (b)

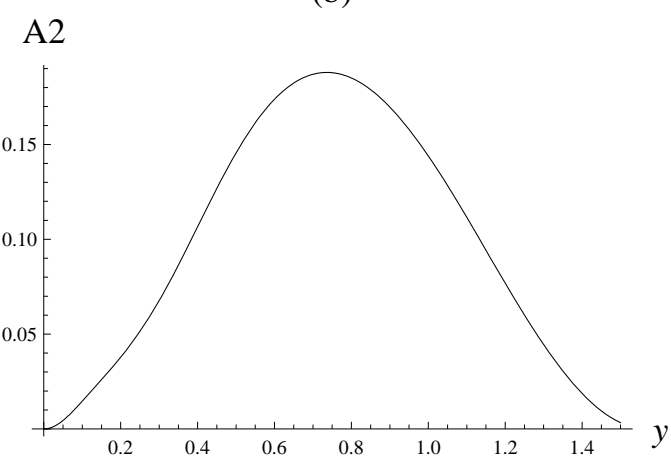

(d)

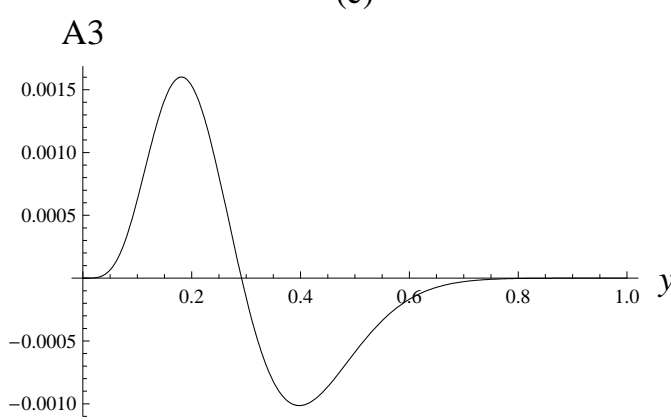

A4

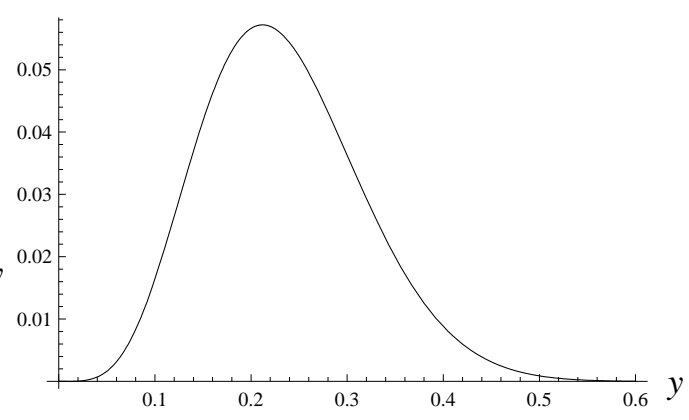

Figure 8: Profiles of the four individual terms the stability quantity $A$ comprises of [Eqs. (14)-(19)] for $\epsilon=0.4, \beta=0.01, M_{0}=0.02$ and $n=6$. Stabilization is caused by combination of the term $A_{2}$ related to the variation of the magnetic field perpendicular to the magnetic surfaces and the flow term $A_{4}$. 
(a) $\epsilon=0.4, \beta=0.01, \mathrm{n}=6, B_{\mathrm{z} 0}=0$

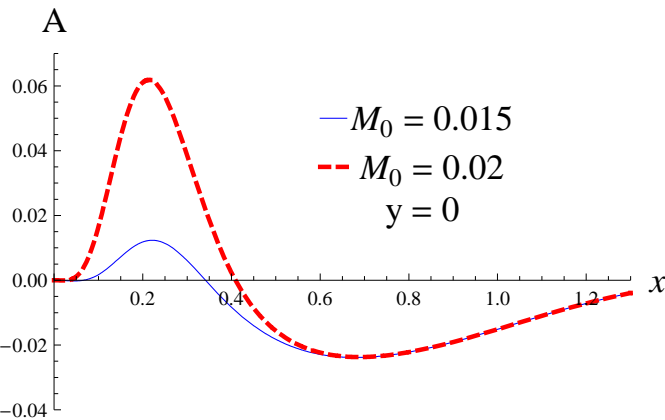

(c) $\beta=0.01, M_{0}=0.02, \mathrm{n}=6, B_{\mathrm{z} 0}=0$

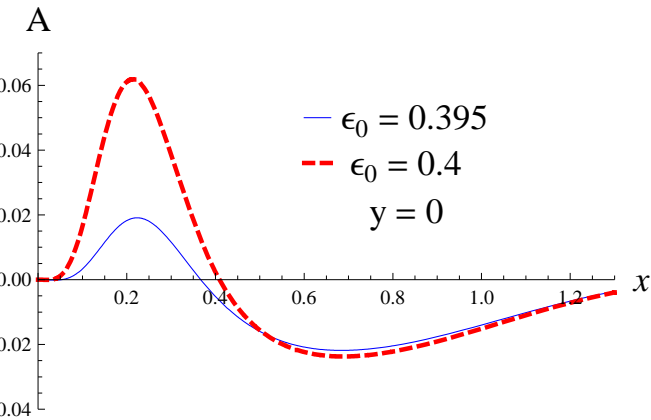

(b) $\epsilon=0.4, \beta=0.01, M_{0}=0.02, B_{\mathrm{z} 0}=0$

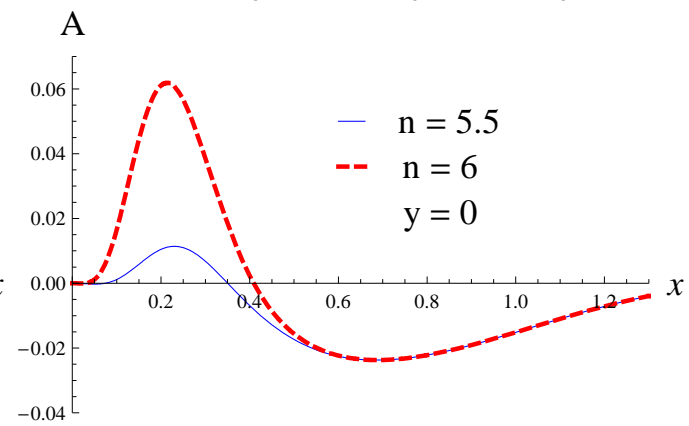

(d) $\epsilon=0.4, M_{0}=0.02, \mathrm{n}=6, B_{\mathrm{z} 0}=0$

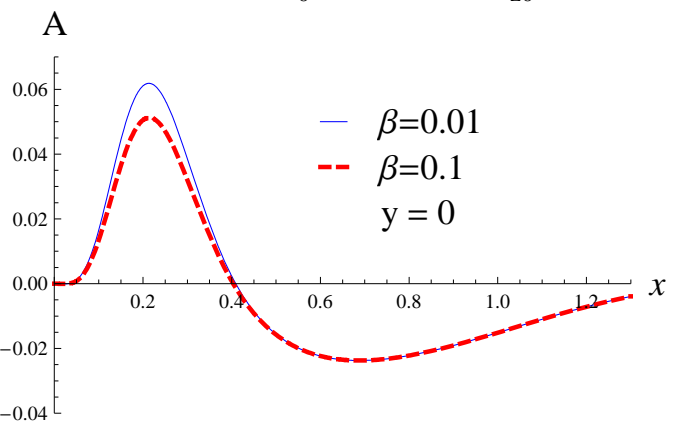

Figure 9: (Color on line) Impact of the flow (a), flow shear (b), island size (c) and thermal pressure (d) in connection with a variation of the parameters $M_{0}, n, \epsilon$, and $\beta$, respectively, on the flow caused stable window associated with $A \geq 0$ for the equilibrium of Fig. 3(a). 
(a) $\epsilon=0.4, \beta=0.01, \mathrm{n}=6, B_{\mathrm{z} 0}=0$

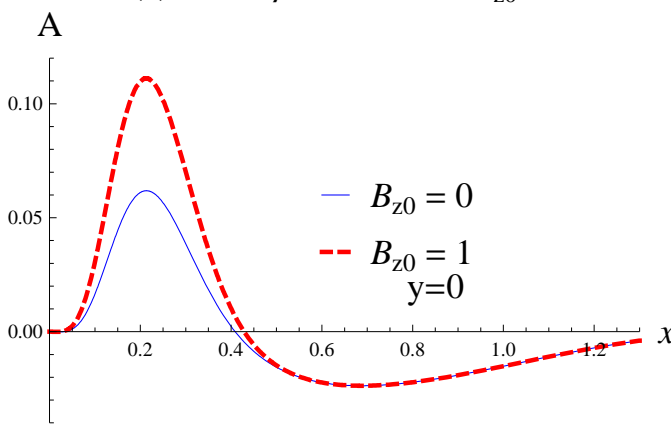

(b) $\epsilon=0.4, \beta=0.6, \mathrm{n}=6, M_{0}=0.02$

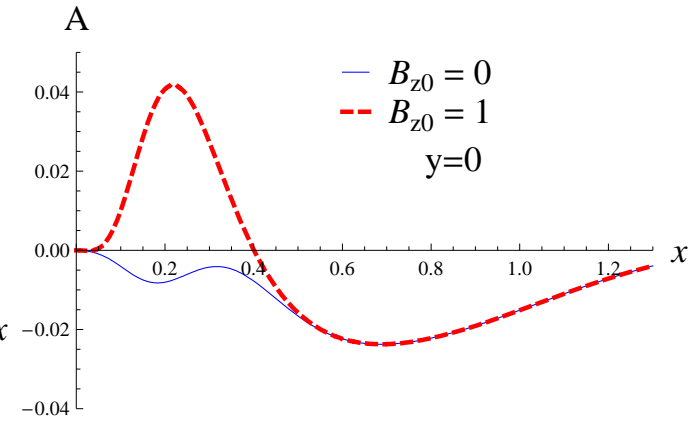

Figure 10: (Color on line) Combined stabilization effect of flow and $B_{z 0}$ : The curve (a) indicates a stabilizing synergism of $B_{z 0}$ and flow for the equilibrium of Fig. 3(a). A stronger synergism of this kind is shown in Fig. 3(b) in which the stability window can not appear by the sole presence of flow. 